\title{
Temporal and Spatial Traffic Analysis Based on Human Mobility for Energy Efficient Cellular Network
}

\author{
Zhigang Li ${ }^{1 *}$, Xin Wang ${ }^{1}$, Junsong Zhang ${ }^{1}$, Wei Huang ${ }^{1}$, and Ye Tian ${ }^{2}$ \\ ${ }^{1}$ School of Computer and Communication Engineering \\ Zhengzhou University of Light Industry, Zhengzhou, 450002, China \\ [e-mail: Lizg.cn@hotmail.com, xinwang_m@163.com, zhangjs2002@foxmail.com,2015109@zzuli.edu.cn] \\ ${ }^{2}$ State Key Laboratory of Networking and Switching Technology \\ Beijing University of Posts and Telecommunications, Beijing, 100876, China \\ [e-mail: yetian@bupt.edu.cn] \\ *Corresponding Author: Zhigang Li
}

Received August 19, 2020; revised December 24, 2020; accepted January 11, 202 1;

published January 31, 2021

\begin{abstract}
With the drastic growth of Information and Communication Technology (ICT) industry, global energy consumption is exponentially increased by mobile communications. The huge energy consumption and increased environmental awareness have triggered great interests on the research of dynamic distribution of cell user and traffic, and then designing the energy efficient cellular network. In this paper, we explore the temporal and spatial characteristics of human mobility and traffic distribution using real data set. The analysis results of cell traffic illustrate the tidal effect in temporal and spatial dimensions and obvious periodic characteristics which can be used to design Base Station (BS) dynamic with sleeping or shut-down strategy. At the same time, we designed a new Cell Zooming and BS cooperation mode. Through simulation experiments, we found that running in this mode can save about $35 \%$ of energy consumption and guarantee the required quality of service.
\end{abstract}

Keywords: Human Mobility, Temporal and Spatial, Traffic Fluctuation, Energy Efficient

This work was supported by the Science and technology project of henan province (Grant No. 192102210296) and the science and technology project of henan province (Grant No. 202102210182) in China. 


\section{Introduction}

Currently, it has been known that the Information and ICT industry has experienced a drastic growth during the past few decades, and it continues to grow rapidly. The ICT infrastructure is responsible for $2 \%$ of global carbon emissions and $3 \%$ of the world's annual electrical energy consumption, which is expected to double by 2020 . Every year, $0.2 \%$ of global energy is consumed by mobile communication networks, which shows exponentially increasing energy consuming figures of mobile communication [1]. Janet Light [2] proposes green computing, combining software-defined networking, edge computing and virtualization to achieve an efficient green network solution. Moreover, it has been revealed in [3] that up to $80 \%$ of the energy consumption in a cellular network is attributed to the operations and functionality of the Base Stations (BS) in the Radio Access Network (RAN) while the remaining energy is expended in the switching and core networks.

With the rapid increase of mobile users as well as mobile services, to allow more users to access network, the mobile network usually deploys network resources in accordance with the requirement on peak capacity. However, the time of a specific cell lying in the peak traffic state is transient and the distribution of user and traffic in time and space shows non-uniformity, which makes a huge amount of communities in a state of excessive coverage in quite a long period, and therefore brings more energy consumption and operation cost [4]. So, from this perspective, how to greatly reduce the energy consumed by the BSs has been paid attention by more and more researchers. Consequently, a huge number of plans on designing energy efficient cellular network have emerged. Related works including [5-9], they brought about using dynamic BS operations to reduce the energy consumption, allowing the system to entirely switch off under-utilized BSs and transfer their loads to neighboring BSs during low traffic periods. Marsan et al. [10] investigated the sleeping strategy according to the temporal traffic variation. The authors in [11] proposed a cell activation mechanism that enables BSs to be activated repulsively according to traffic demands and thus the effective BS density can be scalable for traffic fluctuation. For energy-efficient operation of low power BSs, Niu et al. [12] [13] in proposed a cell zooming mechanism where it was shown that the power consumption can be reduced by means of turning off some BSs and extending the coverage of the other BSs during periods of low traffic demands. In addition, the numerical analysis in the work [14] shows that offloading policy based on the cell range expansion greatly reduces the macrocell traffic, while bringing a small amount of growth for the picocell traffic. Archiman Lahiry et al. [15] proposed a low-cost antenna array system and an adaptive cell densification technology suitable for energy-saving long-term evolution mobile wireless networks. Similarly, in work [16] develop a traffic-aware spatio-temporal model for the contention-based RA analysis in the mIoT network, where the signal-to-noise-plus-interference ratio (SINR) outage and collision events jointly determine the traffic evolution and the RA success probability. S. He et al. [17] proposed a local routing adjustment algorithm. After a new path is found, the local routing adjustment algorithm can be performed to make local adjustments to the selected path to further reduce wireless interference.

The consumption reduction methods mentioned above dynamically deployed BS based on actual traffic demand or low traffic periods without exception. However, they just illustrated the phenomenon of unbalanced cell traffic with no detailed analysis on the specific causes of this traffic behavior. Even [18] uses large scale of user behavior constraints, similarly, it mainly concentrates on the unbalanced traffic distribution of the large scale of users in spatial instead of analyzing the impact of temporal and spatial characteristics of human mobility on traffic fluctuation and the cause of traffic fluctuation. To design a more flexible system-level 
consumption reduction strategy, we need to analyze temporal and spatial characteristics of cell users and the fluctuation characteristics of traffic data in time and space to find the temporal and spatial distribution features of the BSs which in idle running condition.

Following this line of thought, in this paper, we investigate the reason why the traffic in a cellular network is typically unbalanced and fluctuant and it turns out to be the human mobility in time and space like the day-night behavior of users. Many previous works [19, 20] have shown that human trajectories show a high degree of temporal and spatial regularity, and they indicate that there is a potential of $93 \%$ average predictability in human mobility, an exceptionally high value rooted in the inherent regularity of human behavior. This inherent similarity in travel patterns could impact all phenomena driven by human mobility like the design of cellular network in this case.

By analyzing the real dataset which are collected from wireless network at Dartmouth College [21], we analyze the temporal and spatial characteristics of human mobility as well as traffic data such as human's location changes based on time, the locations where traffic data is distributed intensive and so on. Based on these characteristics we can explore a more viable approach for energy efficiency design. In this paper, our main contribution is to exploit the real dataset to analyze the temporal and spatial characteristic of human mobility and its effect on traffic of cellular network. In addition, we confirmed the fact that the cell traffic has the feature of tidal effect in time and space and obvious periodic characteristics, which is used to guide the energy efficiency design in cellular network.

The rest of this paper is organized as follows: In Section II, we give the problem statement for energy efficiency in cellular network. In Section III, we analyze the impact of human mobility on the traffic of cellular network. Section IV consists of the results and discussions on analysis of cellular network traffic which is used to guide the cellular network energy efficient, and we conclude this paper in Section V. In Section V, we conducted energy efficient design and effect analysis and we conclude this paper in Section VI.

\section{Problem Statement for Energy Efficiency}

\subsection{Cellular Network Energy Consumption}

With the development of mobile network technology, the consumption of the energy in the process of cellular network operation and maintenance is increasing year by year. Therefore, how to reduce the energy consumption of the network has aroused widespread concern in the communication industry. With the expansion of the mobile terminal types and functions, the business provided by network becomes increasingly diversified and the deployment of wireless access network BS is more and more intensive. The temporal and spatial distribution of users and business types are presenting increasingly non-uniformity and uncertainty, so network resources are usually provided in accordance with the peak capacity demand in cellular networks, and keep in the non-stop running status in order to ensure the user access to the mobile network at any time and any place. In fact, the length of the peak traffic of a particular network or a particular cell is usually very short, and the traffic is in non-uniform dynamic distribution in space [22]. When the network coverage area is in low load, the resources of BS will not make full use, resulting in a lot of cells in a state of excessive coverage for a long time and wasting a lot of resources.

Especially with the appearance of a large number of smart phones and video business, user demand for peak traffic rate continues to rise, and has burst even more. The emerging data and video broadcast services can often tolerate some delay, so it is not always for the network to 
work in accordance with the peak traffic. Moreover, the rapid popularity of internet of things application can bring amounts of middle and lower rate of business to network, with small but very frequent request volume of business which actually consumes large amounts of energy of BS.

\subsection{Human Mobility and Traffic Fluctuation}

First, we need to analyze the mobile characteristic of human who being as the main producer of traffic. The work [23] studied the mobile pattern of human with the aim of investigating the behavior patterns of user groups, thus it is not difficult to imagine the certain rules obeyed by most of people travelling in daily life, such as workers between the company and home address, students to and from school and home, etc. Yet there are also occasionally uncertain travel routes for human to take, such as tourism, business trips, etc., but they are just small probability events among relatively stable travel events. Besides, in the work of [24], through analyzing the characteristics of human mobility according to the social attribute characteristic of network, we find the fluctuation of population exists in many places such as commercial areas, office areas, residential areas and there is big difference between the day and night.

According to the above analysis, we can find it is the mobility of human that causes nonuniform distribution of users in time series. Because of certain rules followed by human mobility, the dynamic distribution of uses in the cell and business by the users in the cell in time series can be gained through analyzing the data of human mobile track. Furthermore, it is shown that human mobility will have an impact on mobile traffic fluctuation because the main producer of traffic is human and tidal migration of human will inevitably lead to the fluctuation of traffic. Thus by mining the mobility behavior of human we can find the nonuniform distribution characteristics of traffic presented in time and space in mobile network. The distribution characteristics of traffic can also be obtained by collecting and measuring the operation traffic of mobile wireless communication system. The non-uniform distribution of traffic in time domain and the space was found in mobile network. If we combine the characteristic of human mobility with the fluctuation of traffic, we can find more information which is used to guide the energy efficiency design in cellular network.

\section{Human Mobility Analysis for Cellular Network Traffic}

The previous works [19] have shown that human mobility presents a high degree of temporal and spatial regularity, and human always periodically return to the frequently visited position. The work [20] indicates that there is a potential of $93 \%$ average predictability in user mobility, an exceptionally high value rooted in the inherent regularity of human behavior. Based on the characteristics of human mobility which have the rules to follow, since human is the main producer of traffic so human mobility is bound to cause the traffic fluctuation. Therefore, we exploit the real dataset to analyze the temporal and spatial characteristic of the human mobility. In this paper, we used wireless access point network dataset of the Dartmouth campus [21], which has been collected over five years and has over 190 buildings on 200 acres, about 5500 students and 1200 faculties and during our study there were approximately 3200-3300 undergraduates on campus. There are nearly 500 access points (APs) were installed to cover most of the campus. They used three techniques to collect data which are syslog events, SNMP polls, and network sniffers (tcpdump) respectively. On the basis of the campus construction plan, the campus is divided into six main types of area, they are academic (Acad), administrative (Adm), athletic (Athl), library (Lib), social (Soc) and residential (Res) respectively. The Soc area includes communal centers, eating areas etc. Through the partition 
of distribution, we divide the wireless access point networks into corresponding six heterogeneous cells which are based on the regional coverage area as a macrocell, microcell or picocell. We analyze one year data and find the inherent laws it presented follow high uniformity. In this article, the inherent laws that illustrated and discussed by using a small amount of simulation results were shown in Section III and Section IV.

We first study the temporal and spatial distribution of the users which belong to the six heterogeneous cells. We randomly selected a working day mobile trajectory from 0:00 AM to 24:00 PM. As shown in Fig. 1, we averagely divided it into 12 times quantum a day which is expressed in T1-T12. As we locate the positions of users in every two hours, the position which users stay for the longest in these two hours is selected as users' coordinate. We use six different colors to represent six corresponding cells of users respectively in Fig. 1.

From the figure we find that the number of users changes as the time passes. Especially in Fig. 1(T1) and Fig. 1(T2) the nodes are fewer because the number of active users decreases during the period of deep in the night. But from the beginning of Fig. 1(T3), nodes started to rise to a higher level, and the number of users becomes less at the very beginning of evening in Fig. 1(T11). From the perspective of cell, taking Acad and Lib as an example, we can see that the Fig. 1(T1), (T2), and (T3) are almost all blue nodes; only a small number of users are in the presence of Acad and Lib cells because after nightfall the people returned to the residence cell. The same rule, from Fig. 1(T3) users of these two cells began to reach a higher level, and after Fig. 1(T9) the number of users began to reduce significantly because users at night almost all left the Acad and the Lib cells to return to the residence cell. The similar phenomenon also exists in the other four cells.
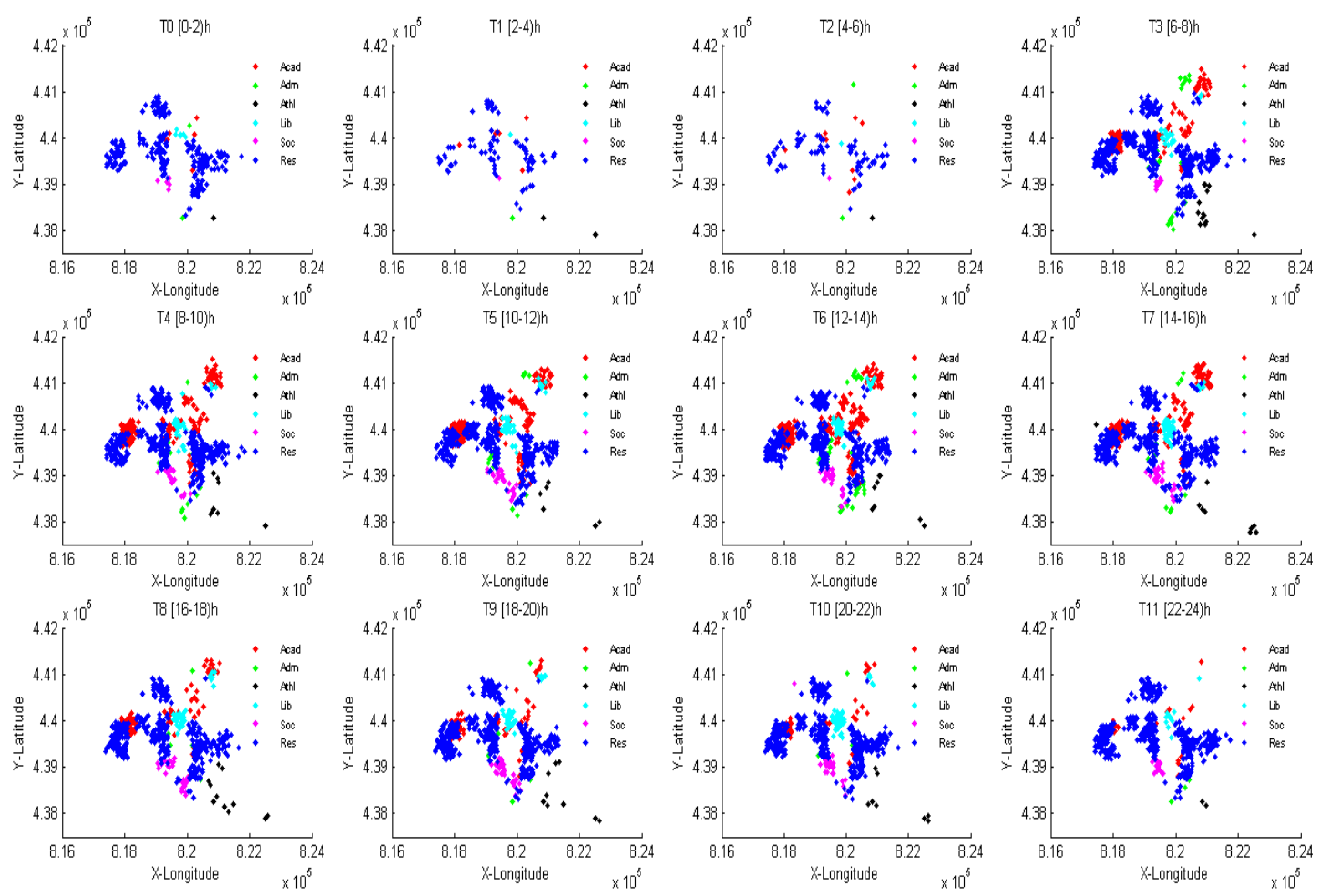

Fig. 1. Temporal and spatial trajectory distribution in the period of a day 
Through the above analysis we can find that human mobility has a high degree of regularity in time and space, so large-scale of users' trajectory can be used to predict the temporal and spatial distribution of users. As traffic distribution in time and space be predicted which according to the human mobility, in turn, we can design the efficient cellular network energy efficient solutions.

\section{Traffic Fluctuation Analysis for Energy Efficiency}

In Section III, we analyze the temporal and spatial mobility characteristic of human, which also has impact on the traffic fluctuation in the cell considering human as the main producer of traffic in cellular network. In this section, we still divided into six cells to analyze the amount of traffic. We simulate the traffic of the cell according to the number of users and the length of reside time in cell. The characteristic of traffic on the basis of time and space dimension on a month is shown in Fig. 2. The cell axes corresponding is the Acad, Adm, Athl, Lib, Soc, and Res cells respectively. We can observe that all cells show the same characteristic which is in a state of the lowest traffic of the day between 02:00:00 and 05:00:00, nevertheless, the time that traffic used to maintain a higher level of traffic is nearly ten hours during the day. And then as night the traffic began to reduce gradually, it is verified that the traffic of cell followed the tidal phenomena. We can see from the picture that the traffic in the Res and Acad cells is higher comparing with other cells during the day. It is because we take the students as the main body of campus data and students often learn in class during the day and stay in the accommodation area in other time.

We also found that the traffic of Acad cell shows obvious periodicity which reaches a peak every 12 hours. The reason is that the number of users in Acad cell during the day time is high, at the same time they all stay for a long time, so the traffic also grows naturally. In the evening, almost no user exists in Acad cell, so the traffic is near to zero. In addition, we found the traffic of Acad cell decreased significantly in the last two days of the week which is due to fewer courses and a few students access to the Acad area. It is not difficult to find that the same property of periodicity exists in other five cells. The traffic of Res cell is always much higher than that in other cells. It implies that there are a lot of students staying in the dormitory most of the time. The number of users entered the Adm, Athl and Lib cells is less and they all stay for a short time, which results in the low traffic of those cells all the time.

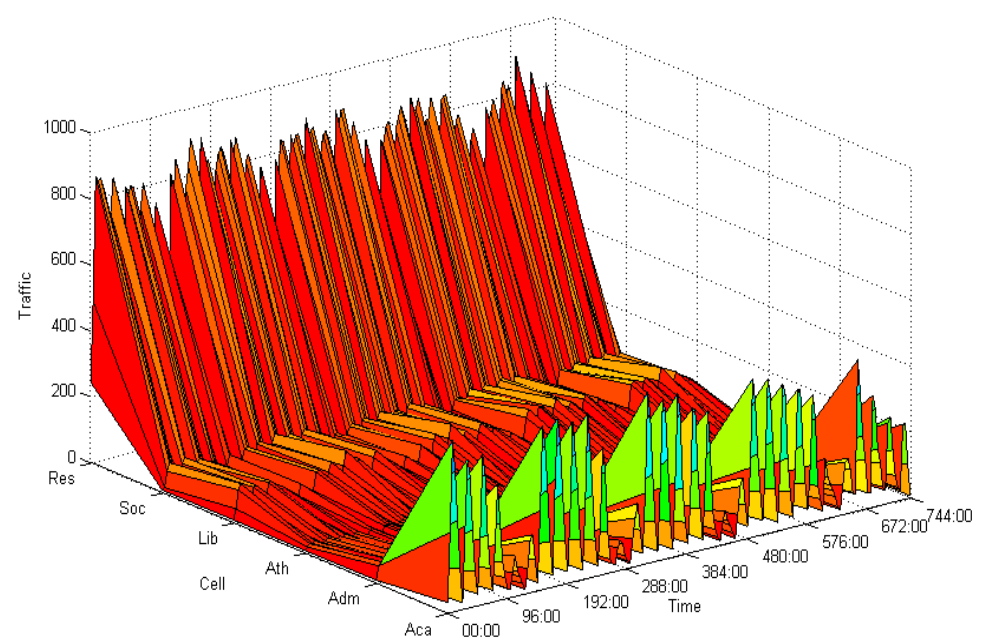

Fig. 2. Temporal and spatial traffic distribution in the period of a month 
Additionally, we find most of the cells are in a state of low traffic operation in most of the time and the cell traffic at peak state time is transient which are due to tidal effects and periodicity, so result in a lot of energy consumption. According to the above findings, we can find that the traffic is non-uniformly distributed in cell and it fluctuates is based on time and space, so we can design efficient and energy efficient cellular network solutions on the basis of temporal and spatial traffic fluctuation characteristic. It also means that we can make use of the tidal effect and periodically of traffic to design BS with dynamic sleeping or shut-down strategy.

\section{Energy Efficient Design and Effect Analysis}

As mentioned in reference [25], the BS has the highest proportion of energy consumption in the cellular network, while the power amplifier occupies a significant proportion of the BS's energy consumption, followed by Air conditioning, Signal Processing and Power Supply. Due to the path loss, it needs enough power to reach the long-distance terminal, but the higher the degree of power amplification, the lower the efficiency. Advanced power amplifiers and new power management schemes need to be designed to adapt to different flow load variations and to adapt energy consumption to actual performance. Therefore, we will reduce the coverage area by appropriately adjusting the transmitting power of the BS, that is, the cell scaling mechanism, to save the energy consumption of the cellular network.

\subsection{Cell Zooming and BS Cooperation Mechanism}

In our work, the cell scaling mechanism is designed according to the fluctuation of cell traffic, and the cell is scaled proportionally according to the level of traffic. The corresponding scaling mechanism is that the power is dynamically reduced or even turned off completely. When some BSs choose to reduce the transmission power, the corresponding traffic load is transferred to the neighboring BSs that remain active, which will inevitably reduce the energy consumption of network operations. Since the traffic demand is much lower than the peak time most of the time, the active BS can easily accommodate the additional traffic load. Therefore, we assume that this operation will not result in a significant increase in call blocking probability or higher interference levels. At this time, when some BSs reduce power or shut down, the network is limited by coverage rather than network capacity.

First, assume that the coverage area of a BS is a hexagonal area with a radius R. Each cell has a BS with multiple antennas located in the center of the cell. We use $h_{k}^{(m)}$. to represent the channel fading index between the m antenna of the $\mathrm{kBS},\left|h_{k}^{(m)}\right|^{2}$ is a cyclically symmetric complex Gaussian random variable with zero mean and variance. The variance is shown in (1):

$$
\sigma_{k}^{2}=\frac{G}{d_{k}^{\alpha}}
$$

Where $d_{k}$ represents the distance from the user to the kth BS, $\alpha$ represents the path loss index.

$G$ is the normalized gain coefficient. Suppose $h_{k}^{(m)}$ is not related to $(k, m)$ and channel state information is available at the BS.

In determining which cell to reduce power or turn off, the optimal solution using integer programming has been proved to be a NP-Hard problem [26, 27]. On the basis of referring to 
two cell shutdown methods, we call these two cell zoom modes I and II respectively, and propose a more efficient method in a specific environment Cell scaling method III. Fig. 3 shows scaling mode III. They scale 1 out of 3 BSs, 2 out of 4 BSs and 2 out of 3 BSs. We use $\beta$ to represent the proportion of the number of BSs scaled or even closed in the three modes in the total number of BSs, from which can be obtained $\beta_{I} \in[0,1 / 3], \beta_{I I} \in[0,1 / 2]$ and $\beta_{I I I} \in[0,2 / 3]$.
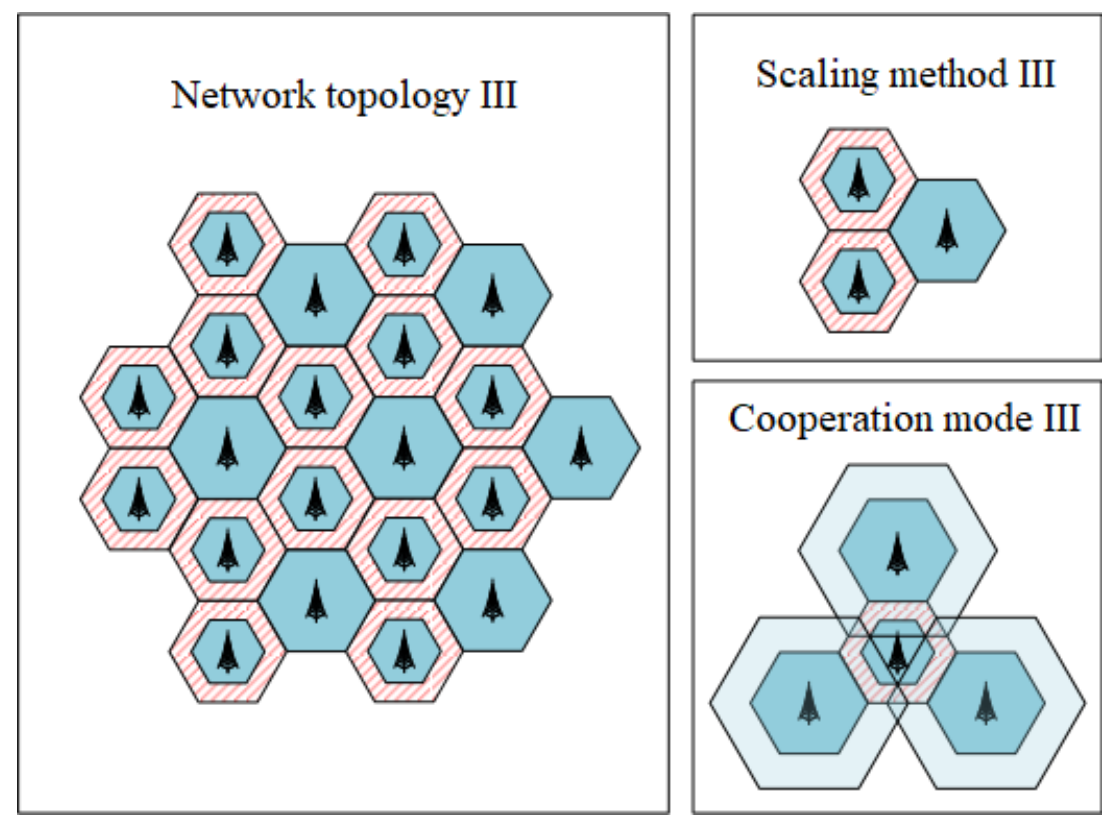

Fig. 3. Scaling mode III: Scaling 2 of 3 BSs

Extend coverage to users located in the service area of the scaled BS to compensate for Signal to Noise Ratio (SNR) loss caused by increasing the distance between the BS and the user. Neighboring BSs will use cooperative communication and power control. SNR is the ratio of the measured signal to noise at the receiving end of the communication system, the signal in this refers to the electronic signal that comes from outside the device and needs to be processed through this device, noise refers to an irregular extra signal that does not exist in the original signal generated by the device, and this kind of signal does not change with the change of the original signal. We restrict the group cooperation BSs to only reduce the power of the directly adjacent BSs or turn off the cells. Among them, the BS scaling mode III corresponding BS cooperation mode is shown in Fig. 3.

In BS scaling mode, when a BS reduces power or shuts down, it will release its channel resources to adjacent active BS. Then the adjacent active BS use the resources obtained from the scaled BS to collaborate to serve the users of the scaled cell. From the network topology, there are only a few BSs with expanded coverage in a number of adjacent cells, and the BSs with expanded coverage expand the transmission power to provide user services.

If the user is in a cell scaled by a BS, we will calculate the SNR of the downlink and the uplink respectively. The downlink and uplink SNR in collaboration mode are calculated as follows: 1) Downlink: since channel state information is available at the BS, the optimal cooperative transmission mechanism is cooperative beamforming. The SNR on the receiving device is shown in (2). 


$$
S N R=\sum_{k=1, m=1}^{K, M} \frac{\left|h_{k}^{(m)}\right|^{2} P_{B S}(1-\beta)}{N_{U E}}
$$

Where $P_{B S}$ is the transmission power of all BSs, $N_{U E}$ is the noise power of user equipment (UE), $K$ represents the number of all BSs. 2) Uplink: considering that channel state information is available at the BS, maximum ratio combining [28] is the best choice for BS cooperation. The SNR at the receiving BS is shown in (3).

$$
S N R=\sum_{k=1, m=1}^{K, M} \frac{P_{U E}\left|h_{k}^{(m)}\right|^{2}}{N_{k}^{(m)}}
$$

Where $P_{U E}$ is the transmission power of UE, $N_{k}^{(m)}$ is the noise power of the $\mathrm{m}$ antenna of the k BS. For simplicity, it is further assumed that all k and $\mathrm{m}, N_{k}^{(m)}$ are the same, that is $N_{k}^{(m)}=N_{B S}$ so:

$$
S N R=\frac{P_{U E}}{N_{B S}} \sum_{k=1, m=1}^{K, M}\left|h_{k}^{(m)}\right|^{2}
$$

\subsection{Interrupt Probability Analysis}

In our work, we use outage probability as an index to measure the quality of service of users. Given a specific SNR threshold $\gamma_{0}$, an interruption occurs when the instantaneous SNR is lower than this threshold. In order to ensure the quality of service of all users in the BS scaling cell, the average quality of service index of a space is not enough. Since a user is anywhere in the cell, we will focus on the quality of service when the user is at the worst transmission and reception location in the cell.

First of all, let's locate the worst location in the scaling cell, the number and location of known active BSs. Fit their geographical location in the two dimensional coordinate system, and mark a pair of coordinates for the BS and the user location respectively. We assign to cooperative BSs and the user's specific coordinate basis:

$$
B S_{1}=\left(x_{1}, y_{1}\right), \ldots, B S_{k}=\left(x_{k}, y_{k}\right) ; \quad U E=(x, y) \text {. }
$$

Therefore, the distance between $U E$ and $B S_{k}$ is:

$$
d_{k}=\left[\left(x-x_{k}\right)^{2}-\left(y-y_{k}\right)^{2}\right]^{\frac{1}{2}}
$$

To determine the worst location, we will only consider the impact of the average path loss of the decaying component: 


$$
E\left[\sum_{k=1, m=1}^{K, M}\left|h_{k}^{(m)}\right|^{2}\right]=M \sum_{k=1}^{K} \frac{G}{d_{k}^{\alpha}}
$$

Where $K$ represents the number of cooperative BSs and $M$ represents the number of antennas of each BS. Therefore, determining the worst position in the closed hexagon region is equivalent to solving the minimization problem of the following constraint:

$$
\begin{array}{ll}
f(x, y) & =\sum_{k=1}^{K} \frac{1}{\left[\left(x-x_{k}\right)^{2}+\left(y-y_{k}\right)^{2}\right]^{\frac{\alpha}{2}}} \\
\text { s.t. } \quad-\sqrt{3} R \leq \sqrt{3} x+y \leq \sqrt{3} R ; \\
-\sqrt{3} R \leq \sqrt{3} x-y \leq \sqrt{3} R ; \\
-\frac{\sqrt{3}}{2} R \leq y \leq \frac{\sqrt{3}}{2} R
\end{array}
$$

In the three different cooperation modes mentioned above, the worst UE position is shown in Fig. 4, where the pentagonal star represents the worst UE position.
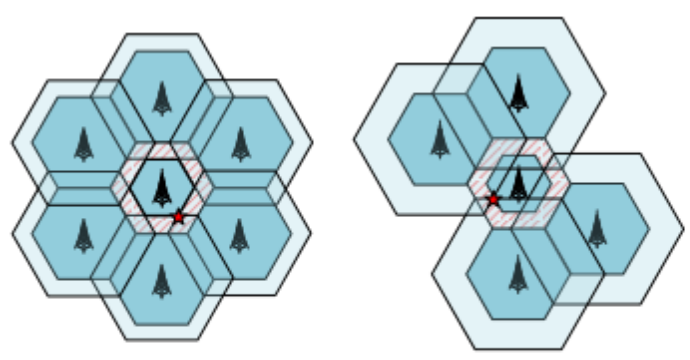

Cooperation mode I Cooperation mode II
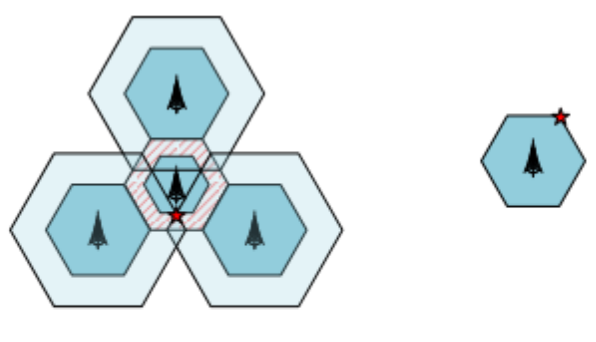

Cooperation mode III Traditional mode

Fig. 4. Worst case UE location

Next we analyze the user's interrupt probability in the BS cooperation mode. Since the downlink and upline transmission scenarios have similar SNR expressions in (2) and (4), our analysis uses the following formula uniformly:

$$
S N R=\sum_{k=1, m=1}^{K, M} \frac{P}{N}\left|h_{k}^{(m)}\right|^{2}
$$

Where $N$ represents the noise power of the receiver and $P$ represents the transmitted power. On the downlink $P=P_{B S}(1-\beta)$.

As shown in Fig. 4, the worst-case UE location in cooperative mode $I$ is the smallest distance from the cooperative BS, and the worst-case UE location in cooperative modes II and III is slightly larger than the cooperative BS I, and has the same distance. However, the distance from the worst UE position to the cooperative BS when the cell is fully scaled to close $d_{I}=d_{I I}=d_{I I I}=\sqrt{3} R$, this geometric arrangement has the same channel gain variance 
$\sigma_{k}^{2}=G /(\sqrt{3} R)^{\alpha}, k=1, \ldots, K$. In cooperative mode I, when cell scaling does not reach the off state, the channel gain variance $\sigma_{k}^{2}=G /\left(\sqrt{\mu^{2}-3 \mu+3} R\right)^{\alpha}$. Where $\mu$ represents the scaling ratio of a single cell. So (9) can be expressed as:

$$
S N R=\frac{P}{N} \frac{\sigma_{k}^{2}}{2} \sum_{k=1, m=1}^{K, M} \frac{2}{\sigma_{k}^{2}}\left|h_{k}^{(m)}\right|^{2}
$$

Since $W \square \sum_{k=1, m=1}^{K, M} \frac{2}{\sigma_{k}^{2}}\left|h_{k}^{(m)}\right|^{2}$ is a central chi-square random variable with $2 K M$ degree of freedom, the cumulative distribution probability of $P_{r}[W \leq \omega]$ is shown in (11):

$$
P_{r}[W \leq \omega]=\frac{\gamma\left(M K, \frac{\omega}{2}\right)}{(M K-1) !}
$$

Where the $\gamma$ function is $\gamma(s, x)=\int_{0}^{x} t^{s-1} e^{-t} d t$. Therefore, the interrupt probability can be expressed as:

$$
P_{\text {out }}=P_{r}\left[S N R \leq \gamma_{0}\right]=\frac{\gamma\left(M K, \frac{\gamma_{0} N\left(\sqrt{\mu^{2}-3 \mu+3} R\right)^{\alpha}}{P G}\right)}{(M K-1) !}
$$

In cooperation mode II and III, as shown in Fig. 4, the distance between the user's worst position and the cooperative $\mathrm{BS}$ is $d_{I I}=d_{I I}=\sqrt{3 \mu^{2} / 4-3 \mu / 2+3} R$ after amplification constraint. Therefore, (9) can be expressed as:

$$
S N R=\frac{P}{N} \frac{\sigma_{k}^{2}}{2} \sum_{k=1, m=1}^{K, M} \frac{2}{\sigma_{k}^{2}}\left|h_{k}^{(m)}\right|^{2}
$$

Where $\sigma_{k}^{2}=G /\left(\sqrt{3 \mu^{2} / 4-3 \mu / 2+3} R\right)^{\alpha}$ is also a central chi-square random variable with a $2 K M$ degree of freedom.

Then analyze the quality of service requirements, when shutting down some BSs, compared to all BSs are active, it is necessary to ensure the minimum quality of service of users rather than deterioration. Therefore, we need to ensure that the user's probability of interruption in the worst location of the scaling cell service area can be slightly lower than or equal to the user's probability of interruption in the worst location of the cell during peak operation. Since a user is served by a single BS in the active cell, its worst position is at the edge of the cell, such as the traditional pattern in Fig. 4. The corresponding SNR (9) is a special case of $K=1$. 


$$
S N R=\frac{P}{N} \sum_{m=1}^{M}\left|h_{0}^{(m)}\right|^{2}=+\frac{P \sigma_{0}^{2}}{N 2} \sum_{m=1}^{M} \frac{2}{\sigma_{0}^{2}}\left|h_{0}^{(m)}\right|^{2}
$$

Where $N$ is the noise power, $P$ is the transmission power, $\left|h_{0}^{(m)}\right|^{2}$ is the channel revenue between the user and the $\mathrm{m}$ antenna of the BS, and its variance is $\sigma_{0}^{(m)}=G / R^{\alpha}$.

In the traditional mode without cell scaling, the same $V \square \sum_{m=1}^{M} \frac{2}{\sigma_{0}^{2}}\left|h_{0}^{(m)}\right|^{2}$ is a central chisquare random variable with a degree of freedom of $2 M$. Therefore, the outage probability of the traditional mode without cell scaling can be expressed as follows:

$$
P_{\text {out }}=P_{r}\left[S N R \leq \frac{2 \gamma_{0} N}{P \sigma_{0}^{2}}\right]=\frac{\gamma\left(M, \frac{\gamma_{0} N R^{\alpha}}{P G}\right)}{(M-1) !}
$$

\subsection{Energy Efficient Effect and Numerical Analysis}

In this part, we quantify the energy efficient effect of the energy efficient network operation mechanism described above. The total energy consumption $P_{\text {totle }}$ of a BS can be divided into two main parts. Idle power consumption $P_{\text {idle }}$, which does not depend on network traffic load. This power consumption means that an active BS is in an idle state with no transmission or reception. The transmission power consumption $P_{R F}$ is dependent on the network traffic load. The major component of this consumption is the Radio Frequency (RF) output component. The transmission and reception power consumption of cooperative BSs is very small compared with the traditional uncooperative BSs, so it is assumed that the additional baseband signal processing power consumption can be ignored.

Here $\eta$ is defined as the ratio of $P_{R F}$ to $P_{\text {totle }}$, while $\lambda$ is defined as the ratio of the required total transmission power to the transmission power required for cooperative transmission, and maintains the same minimum quality of service level as the traditional noncooperative operation. During the energy efficient operation, $\beta$ represents the percentage of all cells scaling. The traffic load of scaling BSs is transferred to the remaining normally active $\mathrm{BSs}$, which means that in order to achieve the required quality of service level, $\beta$ will increase during the energy-saving operation and the power saved in the network will be reduced. Therefore, the percentage of power savings $m$ during the energy saving operation can be calculated by (16):

$$
\begin{aligned}
\xi & =1-\frac{\text { Total Consumption in Energy-Efficient Operation }}{\text { Total Consumption in Conventional Operation }} \\
& =\frac{\beta P_{\text {total }}-\beta \lambda P_{R F}}{P_{\text {total }}} \\
& =\beta[1-\eta \lambda]
\end{aligned}
$$


Next, the proposed Cell Zooming strategy and BS cooperation mechanism are used to display some numerical analysis results and energy-saving performance results. Where in $\phi=P / \gamma_{0} N R^{\alpha} / G, \phi$ function represents the standard SNR level of network given according to system parameters, and denominator $\gamma_{0} N R^{\alpha} / G$ is a predefined of a given network, which is fixed in simulation.

Fig. 5 and Fig. 6 show the interruption probability curves of the three cooperation modes, where the path loss index is $\alpha=3.5$ and $\alpha=4$ respectively. A single BS has $M=6$ transmitting antennas. Curve Baseline QoS Requirement represents the traditional mode in which no BSs cooperate and all BSs operate at peak. Fig. 5 and Fig. 6 clearly show the value of interruption probability. When the interruption probability $P_{\text {out }} \in\left(10^{-4}, 10^{-3}\right)$ in the three BS cooperation modes, it is better than that without the BS cooperation mode. Therefore, the user service quality of the BS cooperation mode can be guaranteed.

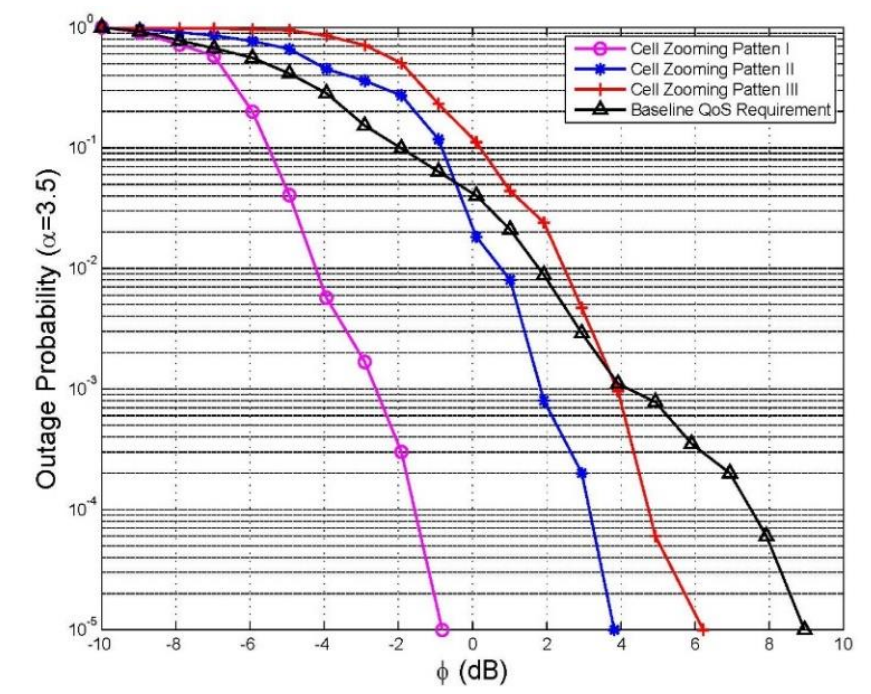

Fig. 5. Interruption probability when the path loss index of scaling mode is 3.5

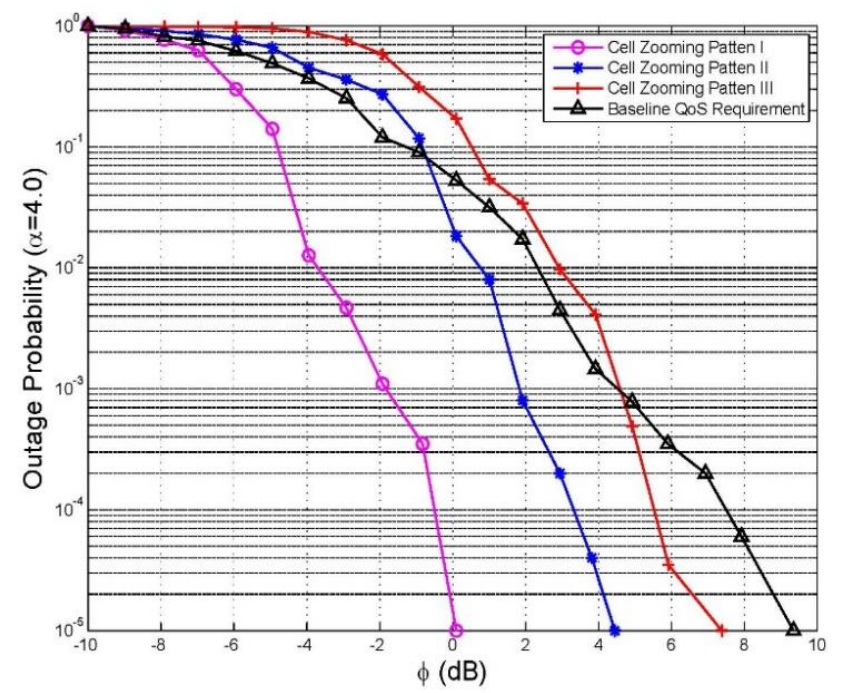

Fig. 6. Interruption probability when the path loss index of scaling mode is 4 
As shown in Fig. 7, using our proposed Cell Zooming mode and BS cooperation mode can significantly reduce energy consumption. Running in this energy saving mode can save about $35 \%$ of energy consumption and can guarantee the required service quality. We can see that the more BS are scaled, the higher the energy savings are when the path loss coefficient is $\alpha=3.5$, On the contrary, when $\alpha=4$, extra transmission power is needed to ensure high quality of service due to the increase of path loss. From Fig. 7, it is not difficult to find that the energy saving effect is not as good as that of $\alpha=3.5$.

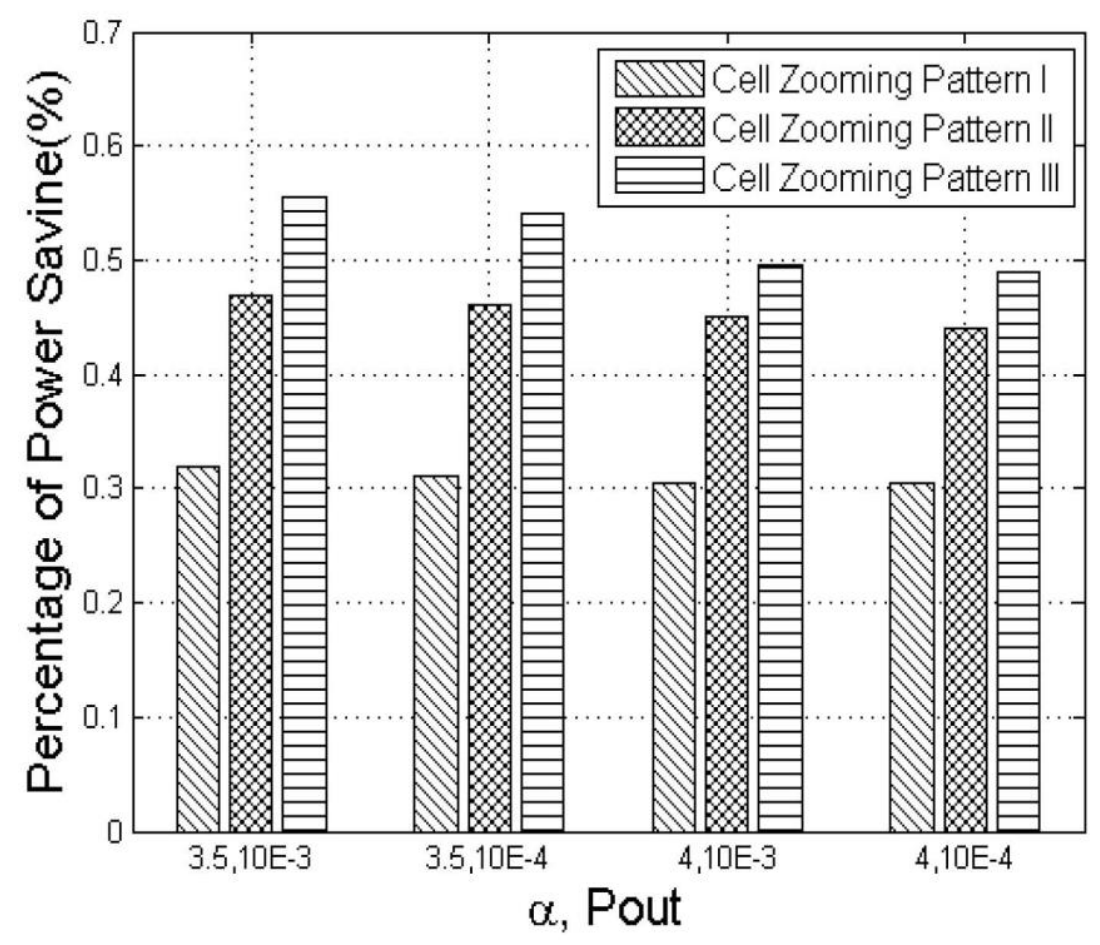

Fig. 7. Energy efficient effect dragram

\section{Conclusion}

The consumption of the energy in the process of cellular network operation and maintenance is increasing year by year. Therefore, reduce the energy consumption of the cellular network has aroused widespread concern in the communication industry. In this paper, we use the real dataset to analyze the temporal and spatial characteristic of human mobility and its effect on traffic of cellular network. We study the potential reason of energy consuming by utilizing the human mobility and non-uniform traffic distributed which is used to guide the energy efficiency design in cellular network. We illustrate the fact that the cell traffic has the feature of tidal effect in time and space and obvious periodic characteristics which can be used to design BS with dynamic sleeping or shut-down strategy. We designed a new cell zooming and BS cooperation mode. Through simulation experiments, we found that running in this mode can save about $35 \%$ of energy consumption and ensure the required quality of service. Future work we will continue to analyze more large-scale data in terms of human mobility and traffic distribution which is used to design an effective scheme for energy saving in cellular network. 


\section{References}

[1] G. Auer, V. Giannini, C. Desset, I. Godor, P. Skillermark, M. Olsson, M. I. Imran, D. Sabella, M. J. Gonzalez, O. Blume, and A. Fehske, "How much energy is needed to run a wireless network?," IEEE Wireless Communications, vol. 18, no. 5, pp. 40-49, Oct. 2011. Article (CrossRef Link)

[2] J. Light, "Green Networking: A Simulation of Energy Efficient Methods," Procedia Computer Science, vol. 171, pp. 1489-1497, Apr. 2020. Article (CrossRef Link)

[3] Z. Niu, S. Zhou, S. D. Zhou, X. F. Zhong, and J. Wang "Energy efficiency and resource optimized hypercellular mobile communication system architecture and its technical challenges," SCIENTIA SINICA Informationis, vol. 42, no. 10, July 2012. Article (CrossRef Link)

[4] F. Richter, A. J. Fehske, and G. P. Fettweis, "Energy Efficiency Aspects of Base Station Deployment Strategies for Cellular Networks," in Proc. of 2009 IEEE $70^{\text {th }}$ Vehicular Technology Conference, pp. 1-5, Sep. 2009. Article (CrossRef Link)

[5] K. Son, H. Kim, Y. Yi, and B. Krishnamachari, "Base station operation and user association mechanisms for energy-delay tradeoffs in green cellular networks," IEEE journal on selected areas in communications, 2011, vol. 29, no. 8, pp. 1525-1536, Sep. 2011. Article (CrossRef Link)

[6] M. A. Marsan and M. Meo, "Energy efficient management of two cellular access networks," ACM SIGMETRICS Performance Evaluation Review, vol. 37, no. 4, Mar. 2010. Article (CrossRef Link)

[7] E. Oh and B. Krishnamachari, "Energy Savings through Dynamic Base Station Switching in Cellular Wireless Access Networks," in Proc. of 2010 IEEE Global Telecommunications Conference(GLOBECOM), pp. 1-5, Dec. 2010. Article (CrossRef Link)

[8] Z. Kuang, G. Li, L. Zhang, H. Zhou, C. Li, and A. Liu, "Energy Efficient Mode Selection, Base Station Selection and Resource Allocation Algorithm in D2D Heterogeneous Networks," Peer-toPeer Networking and Applications, vol. 13, no. 5, pp. 1814-1829, June 2020.

Article (CrossRef Link)

[9] N. Zabetian, A. Mohammadi, and M. Masoudi, "Energy-efficient power allocation for device-todevice communications underlaid cellular networks using stochastic geometry," Transactions on Emerging Telecommunications Technologies, vol. 30, no. 12, Oct. 2019. Article (CrossRef Link)

[10] M. A. Marsan, L. Chiaraviglio, D. Ciullo, and M. Meo, "Optimal Energy Savings in Cellular Access Networks," in Proc. of 2009 IEEE International Conference on Communications Workshops, pp. 1-5, June 2009. Article (CrossRef Link)

[11] S. Cho and W. Choi, "Energy-efficient repulsive cell activation for heterogeneous cellular networks," IEEE Journal on Selected Areas in Communications, vol. 31, no. 5, pp. 870-882, May 2013. Article (CrossRef Link)

[12] X. Weng, D. Cao, and Z. Niu, "Energy-Efficient Cellular Network Planning under Insufficient Cell Zooming," in Proc. of 2011 IEEE 73 ${ }^{\text {rd }}$ Vehicular Technology Conference, pp. 1-5, May 2011. Article (CrossRef Link)

[13] E. Oh, B. Krishnamachari, X. Liu, and Z. Niu, "Toward dynamic energy-efficient operation of cellular network infrastructure," IEEE Communications Magazine, vol. 49, no. 6, pp. 55-61, June 2011. Article (CrossRef Link)

[14] Y. Zhong, T. Q. S. Quek, and X. Ge, "Heterogeneous Cellular Networks with Spatio-Temporal Traffic: Delay Analysis and Scheduling," IEEE Journal on Selected Areas in Communications, vol. 35, no. 6, pp. 1373-1386, June 2017. Article (CrossRef Link)

[15] A. Lahiry, "Analytical Evaluation of an Antenna Array System and Adaptive Cell Densification Technique for Energy Efficient LTE Network," Wireless Personal Communications, vol. 109, pp. 2507-2540, Dec. 2019. Article (CrossRef Link)

[16] Y. Zhong, M. Guoqiang, X. Ge, and F. Zheng, "Spatio-temporal Modeling for Massive and Sporadic Access," IEEE Journal on Selected Areas in Communications, Jan. 2020.

Article (CrossRef Link) 
[17] S. He, K. Xie, K. Xie, C. Xu, and J. Wang, "Interference-aware multisource transmission in multiradio and multichannel wireless network," IEEE Systems Journal, vol. 13, no. 3, pp. 25072518, 2019. Article (CrossRef Link)

[18] Y. Huang, X. Zhang, J. Zhang, J. Tang, Z. Su, and W. Wang, "Energy-efficient design in heterogeneous cellular networks based on large-scale user behavior constraints," IEEE Transactions on Wireless Communications, vol. 13, no. 9, pp. 4746-4757, Sep. 2014. Article (CrossRef Link)

[19] M. C. Gonzalez, A. Cesar, H. A. Hidalgo, and A. L. Barabasi, "Understanding individual human mobility patterns," Nature, vol. 453, pp. 779-782, June 2008. Article (CrossRef Link)

[20] C. Song, Z. Qu, N. Blumm, and A. L. Barabasi, "Limits of predictability in human mobility," Science, vol. 327, no. 5968, pp.1018-1021, Feb. 2010. Article (CrossRef Link)

[21] D. Kotz, T. Henderson, I. Abyzov, and J. Yeo, The Dartmouth Campus Dataset, Sep. 2009. Article (CrossRef Link)

[22] E. Oh, B. Krishnamachari, X. Liu, and Z. Niu, "Toward dynamic energy-efficient operation of cellular network infrastructure," IEEE Communications Magazine, vol. 49, no. 6, pp. 56-61, June 2011. Article (CrossRef Link)

[23] A. S. Cacciapuoti, F. Calabrese, M. Caleffi, G. D. Lorenzo, and L. Paura, "Human-mobility enabled networks in urban environments: Is there any (mobile wireless) small world out there?," Ad Hoc Networks, vol. 10, no. 8, pp. 1520-1531, Nov. 2012. Article (CrossRef Link)

[24] S. Yang, X. Yang, C. Zhang, and E. Spyrou, "Using social network theory for modeling human mobility," IEEE network, vol. 24, no. 5, pp. 6-13, 2010. Article (CrossRef Link)

[25] Z. Hasan, H. Boostanimehr, and V. K. Bhargava, "Green cellular networks: A survey, some research issues and challenges," IEEE Communications surveys \& tutorials, vol. 13, no. 4, pp. 524-540, 2011. Article (CrossRef Link)

[26] R. M. Karp, "Reducibility among combinatorial problems," Complexity of computer computations, pp. 85-103, 1972. Article (CrossRef Link)

[27] R. G. Michael and D. S. Johnson, "Computers and intractability: a guide to the theory of NPcompleteness," Society for Industrial and Applied Mathematics, vol. 24, no. 1, pp. 90-91, 1979. Article (CrossRef Link)

[28] K. Son, E. Oh, and B. Krishnamachari, "Energy-aware hierarchical cell configuration: from deployment to operation," in Proc. of 2011 IEEE Conference on Computer Communications Workshops, pp. 289-294, Apr. 2011. Article (CrossRef Link) 


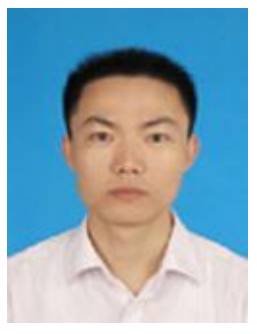

Zhigang Li received the Ph.D. degree from Beijing University of Posts and Telecommunications (BUPT) in 2016. He is currently a research staff of Zhengzhou University of Light Industry (ZZULI), Henan, China. His current research interests include mobile computing, cellular network, VANETs and C-V2X.

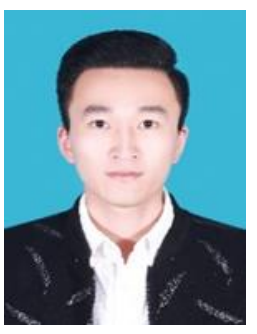

Xin Wang is currently working toward a master's degree with the School of Computer and Communication Engineering, Zhengzhou University of Light Industry, China. His current research interests include VANETs and C-V2X.

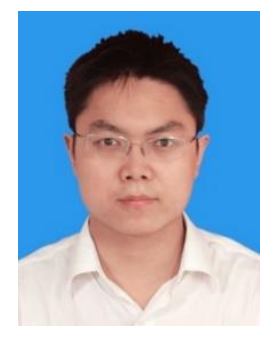

Junsong Zhang received his master's degree in computer software and theory from Zhengzhou University (ZZU) in 2008 and Ph.D. degree in computer science and technology from Beijing University of Posts and Telecommunications (BUPT) in 2014. Dr. Zhang is a lecturer of Zhengzhou University of Light Industry (ZZULI). His research interests include information security and mobile network, etc.

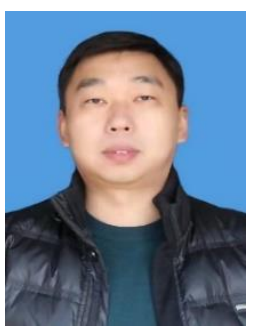

Wei Huang received the Ph.D. degree in pattern recognition and intelligence systems from the Nanjing University of Science and Technology (NUST), Nanjing, China, in 2015. $\mathrm{He}$ is currently a Lecture with the School of Computer and Communication Engineering, Zhengzhou University of Light Industry (ZZULI). His current research interests include pan-sharpening, hyperspectral classification, image processing and machine learning.

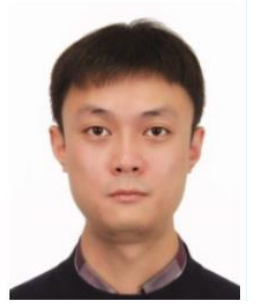

Ye Tian received the B.S. degree from Nanjing University of Posts and Telecommunications (NUPT), Nanjing, China, in 2003 and the M.S. and Ph.D. degrees in computer science and technology from Beijing University of Posts and Telecommunications (BUPT), Beijing, China, in 2007 and 2013, respectively. He is currently a Postdoctoral Researcher with BUPT. He has authored or coauthored over 20 papers. He is the holder of 13 U.S./China patents. His research interests include social network analysis, text mining, and mobile computing. 